\title{
CONGENITAL CUTANEOUS CANDIDIASIS IN A PREMATURE NEONATE. CASE REPORT
}

Daniela Meléndrez-Vásquez ${ }^{1}$, Jose Ruiz-Cabrera ${ }^{1}$, Diana Moreno ${ }^{1}$, and Reinaldo Prieto-Jure ${ }^{1}$

${ }^{1}$ Hospital Universitario Mayor

February 18, 2022

\begin{abstract}
Congenital cutaneous candidiasis is a rare and usually benign disorder that develops within the first week of life. We report a preterm neonate with skin diffuse maculopapular lesions at birth. Candida Albicans was isolated from skin and gastrointestinal fluid culture. Antifungal treatment was initiated with skin lesions resolution.
\end{abstract}

CONGENITAL CUTANEOUS CANDIDIASIS IN A PREMATURE NEONATE: CASE REPORT

Jose Ricardo Ruiz-Cabrera $M D^{1}$, Daniela Meléndrez-Vásquez $\mathrm{MD}^{2^{*}}$, Diana Melissa Moreno $\mathrm{MD}^{2}$. Reinaldo Prieto-Jure $\mathrm{MD}^{3}$

${ }^{1}$ Department of Dermatology, Hospital Universitario Mayor Méderi-Universidad del Rosario Bogotá, Colombia.

${ }^{2}$ School of Medicine and Health Sciences, Hospital Universitario Mayor Méderi-Universidad del Rosario. Bogotá, Colombia.

${ }^{3}$ Department of Neonatology, Hospital Universitario Mayor Méderi-Universidad del Rosario Bogotá, Colombia.

*Correspondence: Daniela Meléndrez-Vásquez, MD, email: daniela.melendrez@urosario.edu.co. Hospital Universitario Mayor Méderi-Universidad del Rosario Bogotá, Colombia. Address: Calle 24 \# 29-45

Key words: Congenital cutaneous candidiasis, Candida Albicans, Case report

Word count 1.097, Number of figures 4, Reference count 11, No supplemental material

Informed consent: Informed consent was obtained from the patient mother with written permission for publication of this case report and associated images. Ethical committee approval.

Competing interests: The funding organization played no role in the report's writing or the decision to submit the report for publication.

\section{CONGENITAL CANDIDIASIS IN A PREMATURE NEONATE. CASE REPORT}

\section{ABSTRACT}

Congenital cutaneous candidiasis is a rare and usually benign disorder that develops within the first week of life. We report a preterm neonate with skin diffuse maculopapular lesions at birth. Candida Albicans was isolated from skin and gastrointestinal fluid culture. Antifungal treatment was initiated with skin lesions resolution. 


\section{KEY WORDS}

Congenital Cutaneous Candidiasis, Candida Albicans, Case report

INTRODUCTION

Congenital cutaneous candidiasis (CCC) is a rare fungal infection caused by ascending intrauterine infection from Candida spp. The clinical presentation takes place within the first 24 hours to the first week of life ${ }^{1-2}$. It is secondary to membrane rupture, chorioamnionitis, or on account of vertical transmission during labor in mothers with Candida infection ${ }^{3}$. Literature around this condition is limited, with only a few case reports. Currently, there are no studies registered in Colombia regarding CCC, therefore this is a pioneering case report in the country.

\section{CASE DESCRIPTION}

A preterm female neonate, gestational age 36 weeks by Ballard score with an unremarkable past prenatal history, vaginal delivery, adequate weight for gestational age $(2730 \mathrm{~g})$. Her mother was a 26 -year old, gravida 1 , para 0 , with a medical history of recurrent urinary tract infections and fungal vaginosis treated during pregnancy. She did not have a vaginal leak, blood, or premature rupture of the membrane before the onset of labor. Spontaneous neonatal adaptation with APGAR scores 7 and 8 at 1 and 5 minutes, respectively. Silverman's scoring system was 3-4. She had low oxygen saturation levels impending respiratory distress therefore, she received non-invasive respiratory support for 24 hours with an adequate response.

During delivery, there was no umbilical cord compromise. Immediately at birth, on examination, multiple erythematous desquamative plaques along with papules and vesicles localized in the back, abdomen, lower and upper extremities, neck, axillary region, retro auricular region, and groin area were observed. Skin lesions had a burning-like dermatitis appearance. There was no palmar or plantar compromise nor mucose and skin appendages involvement. (Figure 1)

Cerebral, abdominal, and cardiac ultrasound were unremarkable. Chest radiography and lumbar puncture were within normal limits. The patient had no ophthalmological compromise. Bloodwork showed a total leukocyte count of $50130 / \mathrm{mm} 3$, neutrophil count of $32720 / \mathrm{m} 3$. Blood cultures were negative after 72 hours and, gastric culture on Sabouraud's dextrose agar and skin potassium hydroxide preparation ( $\mathrm{KOH})$ were positive for Candida Albicans yeast. Further evaluation ruled out immunodeficiency virus, syphilis, and hepatitis $\mathrm{B}$ and $\mathrm{C}$ infection. The study of lymphocyte subpopulations showed normal immunoglobulin, $\mathrm{T}$ lymphocyte, and B lymphocyte levels.

At the neonatal intensive care unit (NICU), the patient received topical Nystatin 100,000 units/g cream four times per day and an intravenous fluconazole loading dose of $25 \mathrm{mg} / \mathrm{kg}$, followed by $12 \mathrm{mg} /$ day IV for seven days. Subsequently, oral fluconazole $12 \mathrm{mg} /$ day and topical Clotrimazole $1 \%$ were continued for 7 days with an adequate response. (Figures 2-4)

\section{DISCUSSION}

CCC is a rare invasive fungal infection of the epidermis and dermis caused by ascending intrauterine infection from Candida spp. CCC has been estimated to occur in $0.1 \%$ of NICU admissions ${ }^{1-3}$. Common risk factors include preterm neonates, gestational age less than 27 weeks, and weight $<1000 \mathrm{~g}^{4}$. In addition, further risk factors are the presence of intrauterine devices, maternal history of cervical cerclage, and invasive maneuvers during delivery ${ }^{5}$. Less common risk factors that may participate CCC are the time of membrane rupture and the mother's diagnosis of fungal vaginosis previously ${ }^{4-6}$. Furthermore, this condition may occur either from vaginal or abdominal delivery ${ }^{4-6}$. Regarding mucocutaneous compromise, manifestations are the result of the aspiration of infected amniotic fluid ${ }^{6}$. In our case, our patient was a preterm neonate with a maternal history of fungal vaginosis.

Clinically, CCC develops within the first week of life, usually within a few hours of birth. It has a heterogeneous presentation that varies according to the following factors: host immune response, number of microorganisms, and time of exposure ${ }^{1-6}$. It typically initiates with diffuse maculopapular exanthema, with 
scaly patches localized in the face, scalp, extensor surfaces of extremities, and umbilical region ${ }^{2}$. Diaper area, palms, and soles are usually spared ${ }^{5}$. Primarily, it begins with erythematous patches that evolve to generalized diffuse papules and pustules, which resolve with desquamation ${ }^{8}$. Other clinical manifestations are paronychia, onychia, and funisitis. In addition, in the context of chorioamnionitis, yellow-white plaques in the proximal umbilical cord could be present ${ }^{10}$. Also, some cases present with skin compromise resembling burning-like dermatitis ${ }^{6}$. Additional clinical features may include pustules in the palmar and plantar region 5 .

Every CCC approach should begin with clinical suspicion. However, a definite diagnosis, including microscopic evaluation, and the culture of Sabouraud dextrose agar from the mucocutaneous lesions, placenta, or umbilical cord, should be assessed ${ }^{5-8}$. The clinical course is benign and auto-limited. Generally, skin lesions resolve within 5-20 days. However, this condition might lead to systemic involvement, the former embrace candida septicemia, meningitis, bronchopneumonia, arthritis, and endocarditis, associated with high mortality rates ${ }^{5}$. Therefore, it is essential to evaluate complications ${ }^{8}$. However, extensive evaluation should be warranted when respiratory distress, positive cultures from blood, urine, and cerebrospinal fluid, leukocytosis with left shift and burning-like dermatitis ${ }^{9-10}$.

Regarding the treatment for CCC, topical Nystatin is the most common treatment, followed by topical Clotrimazole ${ }^{1}$. On the other hand, systemic antifungal therapy has efficacy in neonatal sepsis, weight $<1500$ $\mathrm{g}$, previous treatment with broad-spectrum antibiotics, respiratory distress, positive cultures, and immunodeficiency ${ }^{5-9}$. Management includes non-azole antifungal Amphotericin B, $1 \mathrm{mg} / \mathrm{kg}$ for Amphotericin B deoxycholate, and $5 \mathrm{mg} / \mathrm{kg}$ for lipid-associated Amphotericin B preparations. ${ }^{3-9}$. In addition, 6-12 mg/kg fluconazole is also recommended ${ }^{10}$.

In this case, clinical examination revealed multiple erythematous diffuse maculopapular and scaly patches resembling a burn. $\mathrm{CCC}$ was confirmed with $\mathrm{KOH}$, direct microscopy, culture of the skin, and gastrointestinal fluid culture. Although risk factors for a systemic compromise like respiratory distress, leukocytosis with left shift, and burning-like dermatitis appearance, the patient had an excellent response to treatment with topical and systemic therapy within the first week.

\section{CONCLUSION}

CCC is an invasive infection that can occur in preterm or term neonates with heterogeneous clinical manifestations. Early recognition and prompt diagnosis are essential to initiate treatment to avoid systemic compromise.

Informed consent: Informed consent was obtained from the patient mother with written permission for publication of this case report and associated images

Competing interests: The funding organization played no role in the report's writing or the decision to submit the report for publication.

\section{ORCID}

Daniela Meléndrez-Vásquez: 0000-0003-0018-3196

Diana Melissa Moreno: 0000-0002-2295-5508

Jose Ricardo Ruiz: 0000-0002-1892-8435

Reinaldo Prieto: 0000-0002-7385-3448

\section{REFERENCES}

1. Colantonio S, Hedin E, Li HO, Gavigan G. Management of congenital cutaneous candidiasis in a healthy term baby: A case report.SAGE Open Med Case Rep . 2019;7:2050313X19876707. doi:10.1177/2050313X19876707 
2. Joy Way Bueno SM, Santos Muñoz A, Maldonado I, Larralde M. Onychodystrophy as the only sign of congenital candidiasis.Pediatr Dermatol. 2020 Jan;37(1):159-161. doi: 10.1111/pde.14021

3. Nijamin Romina T, Yarza ML, Grees SA. Candidiasis congénita comunicación de un caso. Dermatol.Argent. 2012;18(3): 225-227

4. Georgescu TA, Lisievici AC, Munteanu O, Furtunescu FL, Bratu OG, Berceanu C, et al. Congenital systemic candidiasis: a comprehensive literature review and meta-analysis of 44 cases. Rom J Morphol Embryol. 2020;61(3):673-680. doi: 10.47162/RJME.61.3.05.

5. Jagtap SA, Saple PP, Dhaliat SB. Congenital cutaneous candidiasis: a rare and unpredictable disease. Indian J Dermatol.2011;56(1):92-3. doi: 10.4103/0019-5154.77564.

6. Miras I, Vierge E, García M, Arruza L, Criado E, Ramos JT, et al. Congenital Cutaneous Candidiasis With Systemic Dissemination in a Preterm Infant. Pediatr Infect Dis J. 2021;40(6):e230-e233. doi: 10.1097/INF.0000000000003080.

7. Wang SM, Hsu CH, Chang JH. Congenital candidiasis. Pediatr Neonatol. 2008;49(3):94-6. doi: 10.1016/S1875-9572(08)60020-0.

8. Tieu KD, Satter EK, Zaleski L, Koehler M. Congenital cutaneous candidiasis in two full-term infants. Pediatr Dermatol.2012;29(4):507-10. doi: 10.1111/j.1525-1470.2011.01468.x. Epub 2011 Sep 9. PMID: 21906141.

9. Aruna C, Seetharam K. Congenital candidiasis. Indian Dermatol Online J. 2014 Nov;5(Suppl 1):S44-7. doi: 10.4103/2229-5178.144531. PMID: 25506564; PMCID: PMC4252951.

10. Fernández-Ruiz M, Mosqueda-Peña R, Pérez-Ayala A, Blázquez-Gamero D. Congenital cutaneous candidiasis associated with maternal peripartum candidemia. Rev Iberoam Micol . 2020;37(2):68-71.

11. Kaufman DA, Coggins SA, Zanelli SA, Weitkamp JH. Congenital Cutaneous Candidiasis: Prompt Systemic Treatment Is Associated With Improved Outcomes in Neonates [published correction appears in Clin Infect Dis. 2017 Oct 15;65(8):1431-1433].

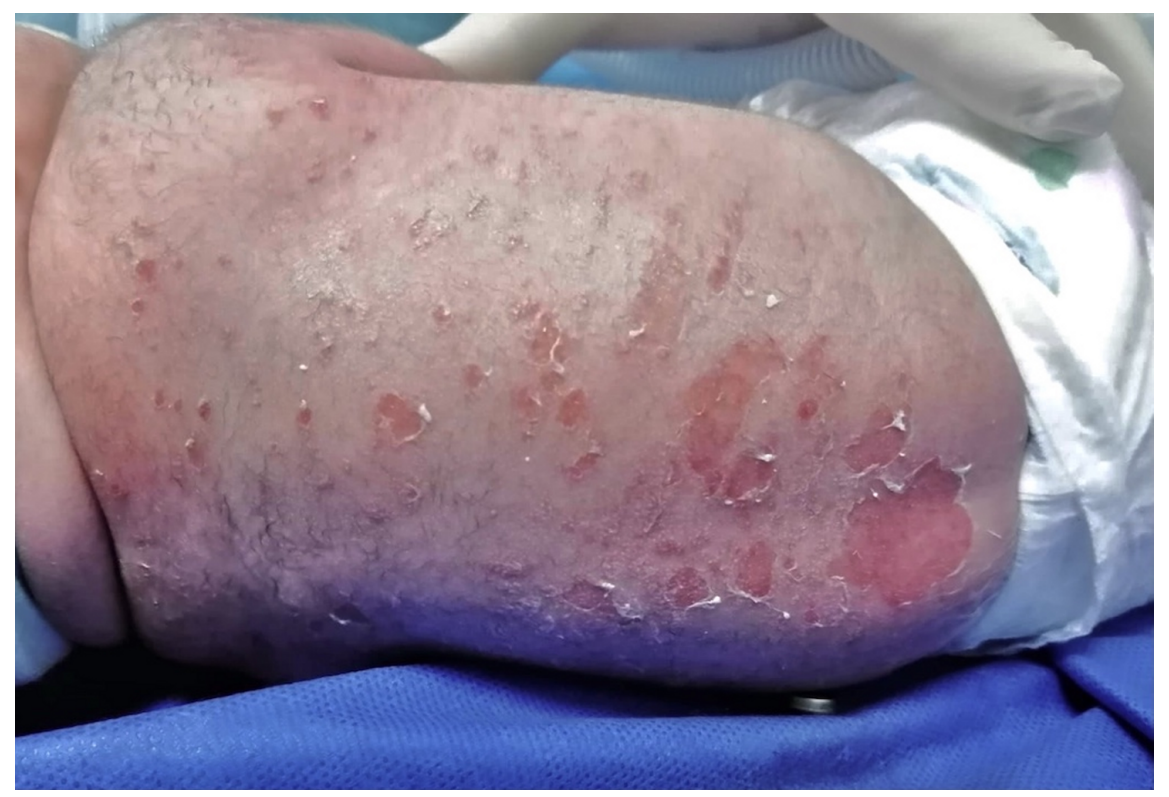



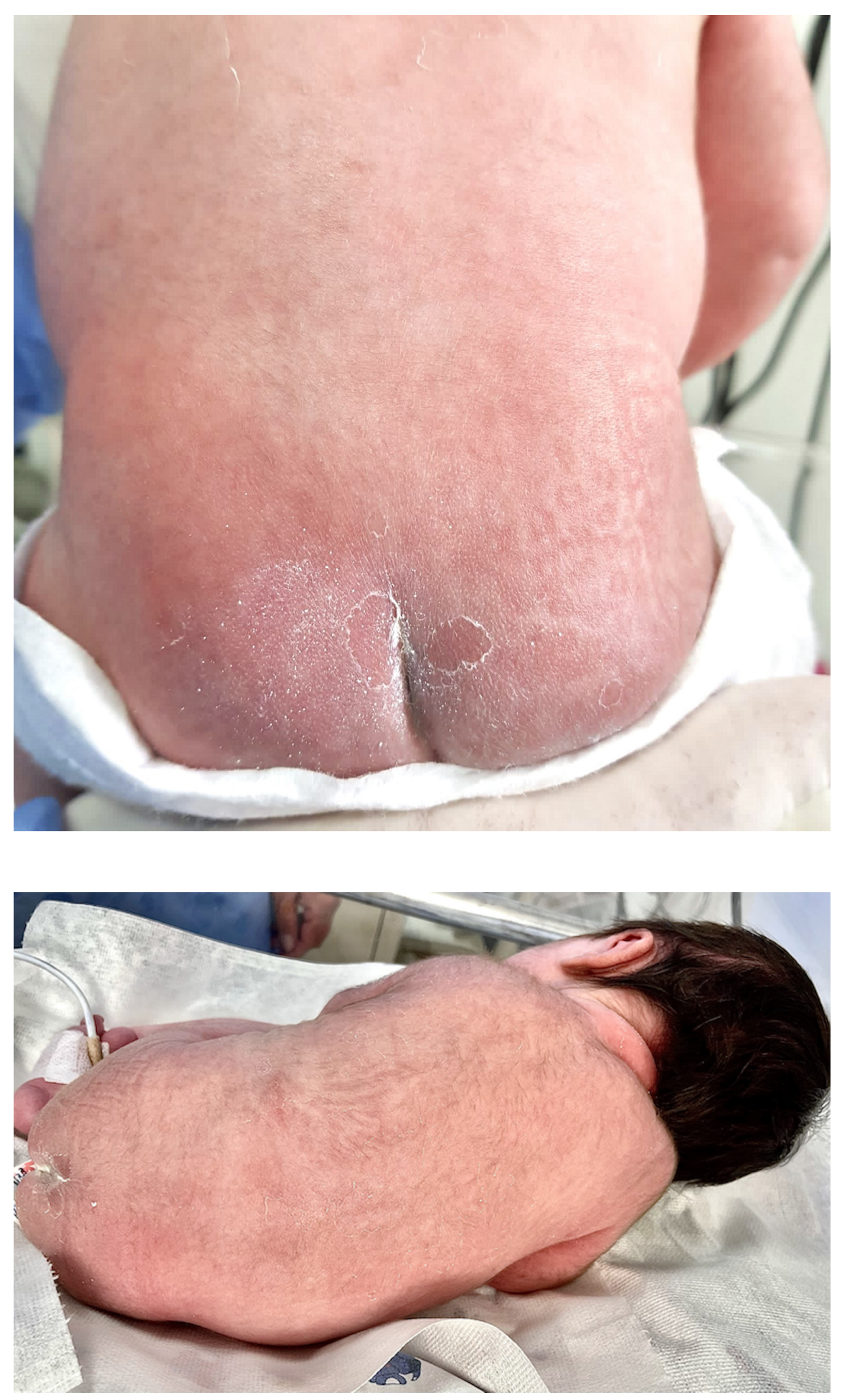


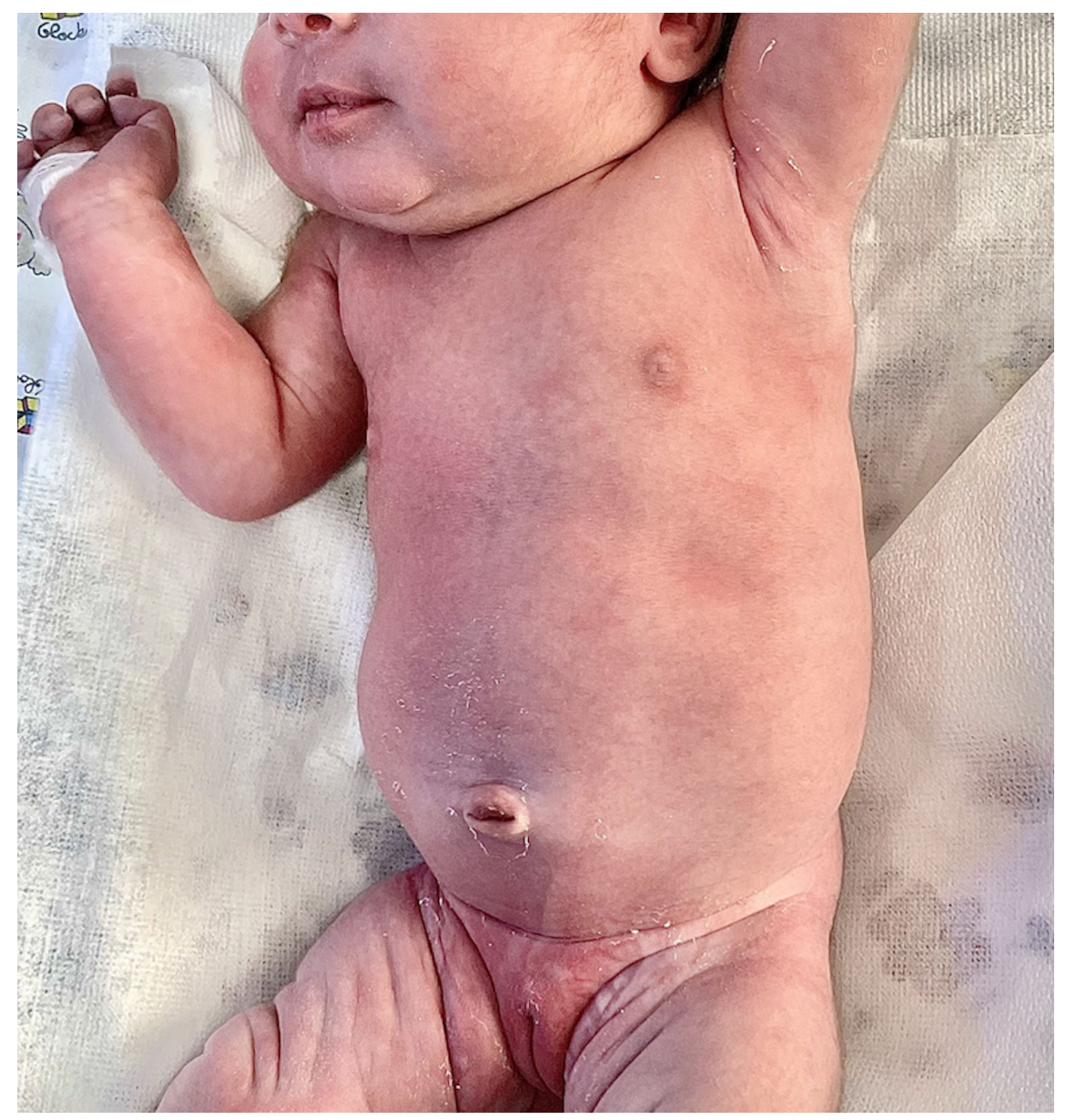

